\title{
Hallmarks of cancer stem cell metabolism
}

\author{
Patricia Sancho*, ${ }^{*}$ David Barneda ${ }^{1}$ and Christopher Heeschen ${ }^{*} 1$ \\ ${ }^{1}$ Centre for Stem Cells in Cancer \& Ageing, Barts Cancer Institute, Queen Mary University of London, London EC1M 6BO, UK
}

Cancer cells adapt cellular metabolism to cope with their high proliferation rate. Instead of primarily using oxidative phosphorylation (OXPHOS), cancer cells use less efficient glycolysis for the production of ATP and building blocks (Warburg effect). However, tumours are not uniform, but rather functionally heterogeneous and harbour a subset of cancer cells with stemness features. Such cancer cells have the ability to repopulate the entire tumour and thus have been termed cancer stem cells (CSCs) or tumour-initiating cells (TICs). As opposed to differentiated bulk tumour cells relying on glycolysis, CSCs show a distinct metabolic phenotype that, depending on the cancer type, can be highly glycolytic or OXPHOS dependent. In either case, mitochondrial function is critical and takes centre stage in CSC functionality. Remaining controversies in this young and emerging research field may be related to CSC isolation techniques and/or the use of less suitable model systems. Still, the apparent dependence of CSCs on mitochondrial function, regardless of their primary metabolic phenotype, represents a previously unrecognised Achilles heel amendable for therapeutic intervention. Elimination of highly chemoresistant CSCs as the root of many cancers via inhibition of mitochondrial function bears the potential to prevent relapse from disease and thus improve patients' long-term outcome.

Cellular Metabolism. In non-transformed, mostly slowly proliferating or even quiescent somatic cells, mitochondria represent the main source of energy production through the tricarboxylic acid (TCA) cycle coupled to oxidative phosphorylation (OXPHOS), which takes place in the mitochondrial matrix. Several carbon fuels such as pyruvate, glutamine and fatty acids can feed into the cycle to produce reducing equivalents (nicotinamide adenine dinucleotide phosphate, NADH; Flavin adenine dinucleotide, $\mathrm{FADH}_{2}$ ) that are subsequently used as electron donors for the electron transport chain (ETC). The transport of electrons across the different complexes of the ETC is coupled to the generation of a proton motive force, used by ATP synthase (complex V) to generate ATP (Chandel, 2014).

Cancer cells, however, are characterised by high proliferation and thus need to adapt their cellular metabolism in order to provide constant support for the increased division rate: rapid ATP generation to maintain energy status, increased biosynthesis of macromolecules and tight regulation of the cellular redox status (Vander Heiden et al, 2009). Moreover, tumour cells must evade the checkpoint controls that under physiological conditions inhibit proliferation in the challenging metabolic conditions regularly found in the tumour microenvironment. Levels of glucose, glutamine and oxygen are spatially and temporally heterogeneous and frequently sparse as compared with conditions in well-perfused organs.
Accordingly, tumour cells reprogramme their metabolic machinery to meet their needs during tumour growth, but also during the changing conditions of the metastatic process.

For this purpose, cancer cells shift from ATP generation via OXPHOS to ATP generation via glycolysis, despite having still sufficient oxygen concentrations in the tumour microenvironment (Warburg effect). As a result, many transformed cells derive a substantial amount of their energy via aerobic glycolysis, which is more rapid than OXPHOS, but also far less efficient in terms of ATP generated per unit of glucose consumed, resulting in an abnormally high rate of glucose uptake. Under these circumstances, glucose is also metabolised through the pentose phosphate pathway (PPP) and other alternative pathways (Vander Heiden et al, 2009), which produce large quantities of reduced NADPH and other macromolecules to generate the necessary building blocks required for sustaining high rates of cellular division.

Cancer stem cells. Over the past decade it has been conclusively demonstrated for leukaemia and various solid cancers that not all malignant cells are functionally equivalent. Convincing evidence now demonstrates that substantial (epi-)genetic heterogeneity exists within each individual tumour. First, multiple subclonal populations of cancer cells are assumed to foster tumour adaptation and therapeutic failure through Darwinian selection.

*Correspondence: Dr P Sancho; E-mail: p.sancho@qmul.ac.uk or Dr C Heeschen; E-mail: c.heeschen@qmul.ac.uk

Received 11 January 2016; revised 15 April 2016; accepted 21 April 2016; published online 24 May 2016 
Second, cancer heterogeneity also exists within each of these subclones, despite their identical genetic background, via the acquisition of stemness features in a subset of cells. The resulting hierarchical organisation of the tumour is vaguely reminiscent of that found in many normal tissues (Figure 1) (Hermann et al, 2007; Malanchi et al, 2012; Visvader and Lindeman, 2012; Garcia-Silva et al, 2013; Miranda-Lorenzo et al, 2014; Chen et al, 2015). At the apex of this hierarchy are populations of cancer stem cells (CSCs) or tumour-initiating cells (TICS), capable of self-renewal, bearing long-term in vivo tumourigenicity as well as generating more differentiated progenies constituting the epigenetically defined intraclonal bulk tumour cells (Hermann et al, 2007).

This new view of intraclonal functional heterogeneity bears the potential to fundamentally change the way we should analyse and treat cancer. To aid this, there will be a need to understand the mechanisms underlying this close relationship between stem cells and their malignant counterparts, which includes their metabolic features. Although CSCs do not necessarily arise from tissue stem cells, these cells have acquired stemness features allowing them to indefinitely self-renew and give rise to their respective differentiated progenies. Epigenetic regulation mimicking, at least in part, normal differentiation contributes to the generation of these hierarchically organised clones that, although sharing common mutation profiles, bear diverse gene expression patterns and functions (Visvader and Lindeman, 2012). Accumulating evidence also suggests striking parallels between mechanisms orchestrating normal embryogenesis and those that invoke tumourigenesis and CSCs in particular (Lonardo et al, 2011). One important feature of (embryonic) stem cells relies in their distinct metabolic phenotype when compared with their differentiated progenies (Folmes et al, 2013) and arising evidence now suggests that CSCs are no exception.

\section{METABOLIC PHENOTYPE OF CSCS}

The metabolic phenotype of CSCs has been the subject of intense investigation over the past years. Interestingly, CSCs have been described as primarily glycolytic or preferentially relying on OXPHOS in a tumour type-dependent manner, but contradictory results for the same tumour entity have also been reported (Table 1). The following paragraphs summarise the key findings for each category.

Evidence for primarily glycolytic CSCs. Originally, it was hypothesised that CSCs should bear a metabolic phenotype reminiscent of normal tissue hierarchy where multipotent stem cells are fundamentally glycolytic, while differentiated somatic cells rely on OXPHOS (Folmes et al, 2012). Similar patterns have been reported for induced pluripotent stem cells (iPS cells), where the reprogramming process is associated with a switch from OXPHOS to a glycolytic programme, which is indeed essential for effective acquisition of a pluripotent state. These findings did not only suggest that the metabolic phenotype and stemness are intrinsically linked, but rather that the cellular metabolism actually controls stemness properties. Thus, it was postulated that activation of the glycolytic programme favours stemness via different mechanism, including enhanced antioxidative capacity, with PPP being most relevant.

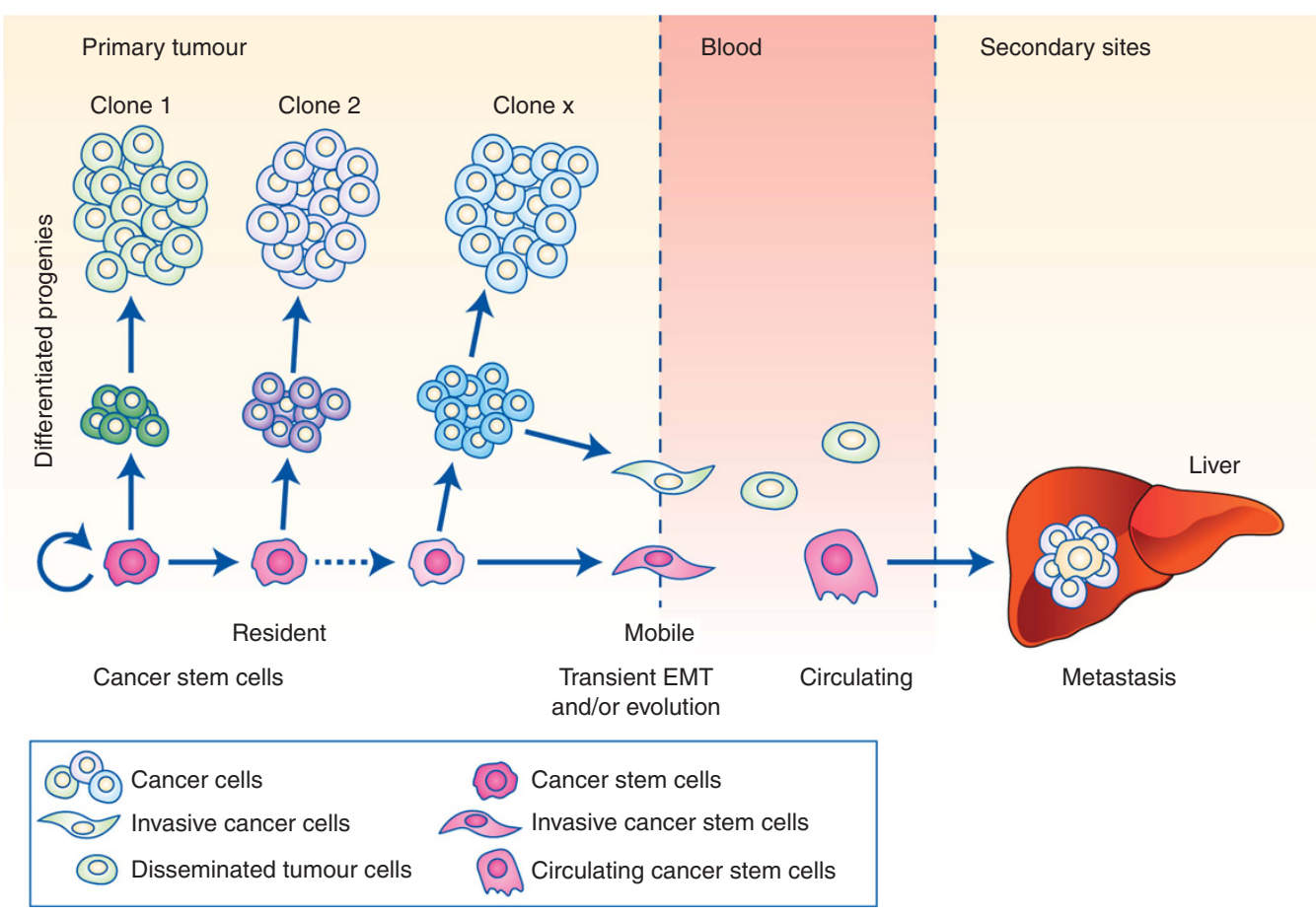

Figure 1. The cancer stem cell concept in cancer progression and metastasis. For various solid cancers it has been shown that intraclonal heterogeneity is formed by CSCs and their differentiated progenies (left) (Visvader and Lindeman, 2012; Miranda-Lorenzo et al, 2014). CSCs are capable of undergoing unlimited cell division while retaining their stem cell identity (self-renewal) and giving rise to more differentiated cells with limited or no tumour-initiating and metastatic capacity, despite their high proliferative capacity. CSCs evolve as the tumour progresses via (epi-) genetic alterations, but also in response to interactions with their niche, leading to diverse CSC subclones with distinct functionality (Sainz et al, 2014, 2015). While both CSCs and differentiated cancer may acquire enhanced mobility, for example, via epithelial-mesenchymal transition (EMT), to date only arising metastatic CSCs have been shown to initiate secondary lesions (Hermann et al, 2007; Malanchi et al, 2012) and are tractable as circulating CSCs in the blood (Clausell-Tormos et al, 2014; Yang et al, 2015) (centre). Importantly, these cells must survive the hostile environment of the blood stream, evade immune surveillance and extravasate at a distant location to form metastatic lesions, rendering the process extremely inefficient (right). 
Table 1. Identified metabolic phenotype for various cancer types (in chronological order)

\begin{tabular}{|c|c|c|c|c|c|c|}
\hline Cancer type & Year & Model/material & Experimental setting & Phenotype & $\begin{array}{l}\text { Sensitivity to } \\
\text { mitochondrial } \\
\text { targeting }\end{array}$ & References \\
\hline Breast & $\begin{array}{l}2010 \\
2013 \\
2013\end{array}$ & $\begin{array}{l}\text { Cell lines } \\
\text { Cell lines } \\
\text { Cell lines }\end{array}$ & $\begin{array}{l}\mathrm{CD} 44^{+} \mathrm{CD} 24^{\text {low }} \\
\mathrm{SP}^{+} \text {sphere-forming cells } \\
\mathrm{CD} 44^{+} \mathrm{CD} 24^{\text {low }} \mathrm{EPCAM}^{+} \text {cells }\end{array}$ & $\begin{array}{l}\text { ND } \\
\text { ND } \\
\text { Glycolytic }\end{array}$ & $\begin{array}{l}\text { Metformin } \\
\text { Niclosamide } \\
\text { ND }\end{array}$ & $\begin{array}{l}\text { (Vazquez-Martin et al, } \\
\text { 2010) } \\
\text { (Wang et al, 2013) } \\
\text { (Dong et al, 2013) }\end{array}$ \\
\hline Lung & 2011 & Cell line & $\mathrm{SP}^{+}$cells, Sphere-forming cells & OXPHOS & ND & (Ye et al, 2011) \\
\hline AML & 2013 & $\begin{array}{l}\text { Primary cultures from human } \\
\text { samples }\end{array}$ & Quiescent ROS ${ }^{\text {low }}$ cells & OXPHOS & ABT-263 & (Lagadinou et al, 2013) \\
\hline Liver & 2015 & Fresh tumours (murine/human) & $\mathrm{CD} 33^{+} \mathrm{CD} 4 \mathrm{f}^{+}$cells & Glycolytic & Paraquat & (Chen et al, 2015) \\
\hline
\end{tabular}

Subsequently, a number of investigations aimed to expand the concept of glycolysis-driven stemness to CSCs and experiments in breast cancer cell lines did support this hypothesis. For example, Dong et al (2013), demonstrated that the metabolic switch from OXPHOS to aerobic glycolysis was essential for the functionality of breast $\mathrm{CD} 44^{+} \mathrm{CD} 24^{\text {low }} \mathrm{EPCAM}^{+} \mathrm{CSCs}$, due to decreased ROS levels. Moreover, glycolysis was also found to be the preferred metabolic programme in radioresistant sphereforming cells in nasopharyngeal carcinoma (Shen et al, 2015) and $\mathrm{CD}_{133}{ }^{+} \mathrm{CD}_{49 \mathrm{f}^{+}}$TICS in hepatocellular carcinoma (Chen et al, 2015). Interestingly, elevated expression of oncogenic MYC was identified as the main driver of stemness for these three cancer types (Gabay et al, 2014), which is in line with findings for iPS cells. While MYC levels did not determine the metabolic wiring of iPS cells, their tumorigenic potential as evidenced by teratoma formation was intrinsically linked to a MYC-driven glycolytic programme (Folmes et al, 2013). Therefore, MYC is a likely candidate for linking glycolysis and stemness, which is intimately related to the tumorigenic potential of iPS cells, and the cancer types listed above.

Evidence for OXPHOS-dependent CSCs. Surprisingly, CSCs in other cancer types have demonstrated OXPHOS as the preferred energy production process. To date, this has been shown convincingly for side population cells in lung cancer (Ye et al, 2011), sphere-forming and CD133 ${ }^{+}$cells for both glioblastoma (Janiszewska et al, 2012) and pancreatic ductal adenocarcinoma (PDAC) (Sancho et al, 2015), and ROS ${ }^{\text {low }}$ quiescent leukaemia stem cells (Lagadinou et al, 2013). Besides glucose, CSCs may also rely on mitochondrial fatty acid oxidation (FAO) for ATP and NADPH generation. Self-renewal in both hematopoietic stem cells and leukaemia-initiating cells appears to be dependent on FAO (Samudio et al, 2010; Ito et al, 2012), a metabolic process that also sustains ATP production and survival in epithelial cancer cells following loss of matrix attachment (Schafer et al, 2009; Carracedo et al, 2012). Interestingly, the pluripotency factor NANOG promoted a highly tumourigenic and chemoresistant metabolic programme in $\mathrm{CD} 33^{+} \mathrm{CD}_{49 \mathrm{f}^{+}}$hepatocellular carcinoma cells by directly inducing the expression of FAO genes (Chen et al, 2016).
Therefore, FAO inhibition offers an additional pharmacological strategy to target mitochondrial metabolism in CSCs (Carracedo et al, 2013).

Although the mechanisms determining the observed OXPHOS phenotype have not yet been fully characterised for all aforementioned tumour types, regulatory proteins of mitochondrial biogenesis and structure could have a crucial role in maintaining stemness properties and functionality (Janiszewska et al, 2012; Sancho et al, 2015). Indeed, findings for PDAC clearly demonstrated that expression of the transcription factor PPARGC1A (PGC-1 $\alpha$ ), a master regulator of mitochondrial biogenesis, in pancreatic $\mathrm{CD} 133^{+}$CSCs was essential for their OXPHOS functionality and, most importantly, self-renewal and maximal in vivo tumorigenic capacity (Sancho et al, 2015). Intriguingly, a MYC-driven glycolytic programme could only be found in more differentiated PDAC cells and overexpression of MYC actually counteracted stemness via negatively controlling PGC- $1 \alpha$ expression. These data seem to challenge the concept for MYC favouring stemness via activation of glycolysis, as demonstrated for iPS cells and other cancer types. However, those apparently contradictory findings could be reconciled in a concept where MYC serves as a general modulator of differentiation, promoting either stemness or differentiation in a context and cell type-dependent manner.

Interestingly, the metabolic rewiring to OXPHOS rendered CSCs in these tumour types resistant to inhibition of glycolysis, which may provide the cells with a higher degree of independency from microenviromental nutrient supply. Indeed, OXPHOS equips CSCs with increased resistance to nutrient deprivation and, in general, to the metabolic austerity characterising many solid tumours. Although OXPHOS operates at a significantly lower rate, it constitutes a far more efficient source for energy generation. Thus, OXPHOS-dependent CSCs may acquire a selective advantage in the context of specific tumour microenvironments, as they make better use of the limited nutrients. In addition, lactate excreted by more differentiated cancer cells that are preferentially running on glycolysis may in return serve as additional fuel for oxidative respiration in cellular subsets that depend on mitochondrial metabolism, that is, CSCs, constituting a metabolic symbiosis system (Nakajima and Van Houten, 2013). 
Controversies and the importance of the utilised model systems. In addition to the above differences in the metabolic phenotype of CSCs derived from various tumour types, contradictory results regarding the CSC metabolic phenotype have also been reported for individual cancer types. Several reasons may account for these unexpected differences. Most importantly, the definition and isolation techniques for CSCs were not uniform across these studies and thus different cellular entities with diverse properties may have been studied. For example, in murine models of ovarian cancer, TICs were obtained following serial in vivo passages (MOSE- $\mathrm{L}_{\mathrm{FFv}}$ ) and were found to be primarily glycolytic (Anderson et al, 2014) as compared with respective parental cells (MOSE-E). On the other hand, $\mathrm{CD} 44^{+} \mathrm{CD} 117^{+}$cells isolated from primary human cultures, either directly from patients with ovarian cancer or following in vivo expansion in immunocompromised mice (PDX models), were OXPHOS-dependent (Pasto et al, 2014). Apart from the potential differences in species, isolated $\mathrm{CD} 44^{+} \mathrm{CD} 117^{+}$cells are unlikely to be comparable to MOSE- $\mathrm{L}_{\mathrm{FFv}}$ cells. The latter may represent the in vivo selection of clones with enhanced aggressiveness, not necessarily presenting stem-related properties.

Even more importantly, first studies on the metabolic phenotypes of CSCs were based on established cancer cell lines. The findings in these studies that the selected putative CSCs are highly glycolytic remains at least debatable as cell lines represent rather homogeneous phenotypes, often with no clear CSC subset based on functional assays (Miranda-Lorenzo et al, 2014) and also lack a suitable tumour microenvironment. Indeed, very recently in lung cancer the metabolic dependencies were found to be different between in vitro and in vivo settings (Davidson et al, 2016), highlighting the importance of the microenvironment for the metabolic phenotype. In line with these observations, certain end products of glycolysis such as high-energy lactanes and ketones released by strongly glycolytic stromal cells promoted the expression of stemness-associated genes and shifted cancer cells towards OXPHOS (Martinez-Outschoorn et al, 2011). Thus, the metabolic phenotype of cancer (stem) cells may heavily depend on microenvironmental conditions and should be studied in fresh tumour samples or, at least, in early passage primary (co-)cultures, where tumour cells are more likely to still present a phenotype reminiscent of that within the tumour. It remains to be determined whether the phenotype of CSCs originally reported for some cancer types will eventually be validated in clinically more relevant models.

Mitochondrial function is crucial for CSC phenotypes. In addition to constituting a major source of ATP for cancer cells, mitochondria participate in controlling multiple signalling pathways, including the release of Cytochrome $\mathrm{C}$ to initiate apoptosis, the release of bioactive ROS and the production of metabolites such as acetyl-CoA regulating protein acetylation (Chandel, 2014). As such, mitochondria also appear to regulate stemness properties, irrespective of the underlying metabolic phenotype in individual cells (Diehn et al, 2009; Lamb et al, 2014; Sancho et al, 2015; Lamb et al, 2015c). Indeed, enhanced mitochondrial biogenesis appears to represent a key factor for CSC functionality in both glycolytic and OXPHOS-dependent CSCS (Janiszewska et al, 2012; De Luca et al, 2015; Sancho et al, 2015). Increased mitochondrial mass, a surrogate marker for elevated mitochondrial biogenesis, can be easily tracked and may identify cells with enhanced self-renewal capacity and chemoresistance (De Luca et al, 2015; Farnie et al, 2015; Lamb et al, 2015a; Sancho et al, 2015) independently of the cancer type. Specifically, in PDAC, the use of this metabolic biomarker allowed to identify a subpopulation of $\mathrm{CD}_{133^{+}}$cells with low mitochondrial mass, but increased metabolic plasticity driven by MYC within the CSC compartment $\left(\mathrm{CD}_{133^{+}}{ }^{+} \mathrm{Mito}^{\text {low }}\right)$ (Sancho et al, 2015). Consequently, these cells showed increased resistance to mitochondrial targeting. However, the metabolic plasticity came at the expense of a reduced self-renewal capacity and in vivo tumorigenic potential, suggesting a delicate balance between stemness and metabolism, thus adding another level of complexity to the metabolic features of CSCs. Whether the state of these two different CSC subpopulations with differential metabolic features and tumorigenic potential is dynamic and cells are able to transition between states, or is hard wired due to distinct genetic backgrounds remains to be elucidated.

\section{TARGETING CELLULAR METABOLISM}

The apparent OXPHOS dependence of CSCs in various tumours and the recently identified role of mitochondria in the regulation of stemness properties (Diehn et al, 2009) suggest that targeting mitochondrial metabolism could be an effective pharmacological strategy for the elimination of CSCs. Moreover, as mitochondria in cancer cells are often altered by mutations in their vulnerable DNA, the pharmacological disruption of certain mitochondrial processes could damage CSCs without affecting healthy tissues also relying on OXPHOS. Pharmacological agents targeting OXPHOS at various levels are currently being explored in preclinical and clinical studies for cancer treatment (Figure 2).

Specifically, targeting mitochondrial OXPHOS could be an effective strategy to eliminate cancer cells, which cannot fully meet their energetic demands by glycolysis, either due to limited availability of glucose in poorly vascularised tumours, glycolysis inhibition by current therapies or restricted metabolic plasticity as observed in $\mathrm{CD}_{133}{ }^{+}$CSCs (Janiszewska et al, 2012; Sancho et al, 2015). Inhibition of mitochondrial respiration by agents blocking ETC complexes selectively induced apoptosis in $\mathrm{CD}_{133^{+}}$versus $\mathrm{CD}_{133^{-}}$cells (Sancho et al, 2015). The fact that such agents are also effective in eliminating primarily glycolytic $\mathrm{CD} 44^{+} \mathrm{CD} 24^{\text {low }}$ cells in breast cancer or side population cells in nasopharyngeal carcinoma (Vazquez-Martin et al, 2010; Shen et al, 2013), highlights the importance of mitochondria for CSCs beyond energy production. In fact, tumour cells displaying mutations impairing TCA cycle or ETC, thus predominantly relying on glycolysis for ATP production, still require active mitochondria for the generation of metabolites from glutamine via reductive carboxylation (Mullen et al, 2012).

Drug screens aiming for the identification of compounds that selectively eliminate CSCs resulted in the selection of several FDAapproved compounds that inhibit mitochondrial activity. For example, the antibiotic salinomycin, which inhibits OXPHOS, was identified in a screen of breast cancer-initiating cells displaying an EMT phenotype and eliminated the CSC gene expression signature in subsequent in vivo studies (Gupta et al, 2009). Remarkably, salinomycin was also selected in an independent screen aimed at targeting colorectal cancer cells in glucose-deprived multicellular tumour spheroids with inner hypoxia (Senkowski et al, 2015), which may more closely reflect the in vivo microenvironment of CSCs in solid tumours. Besides salinomycin, four other compounds were identified (nitazoxanide, niclosamide, closantel and pyrvinium pamoate), all of them inhibiting mitochondrial respiration. Niclosamide, an anti-helmitic drug that uncouples mitochondrial OXPHOS, was also among the selected compounds in two independent screens using side population cells derived from breast and ovarian cancers, respectively (Yo et al, 2012; Wang et al, 2013). Apart from the direct inhibition of mitochondrial complexes, OXPHOS can also be suppressed by inhibitors of mitochondrial translation, for example, using the antibiotic tigecycline, which was identified in a screen using OXPHOS-dependent leukaemia cells (Skrtic et al, 2011). 


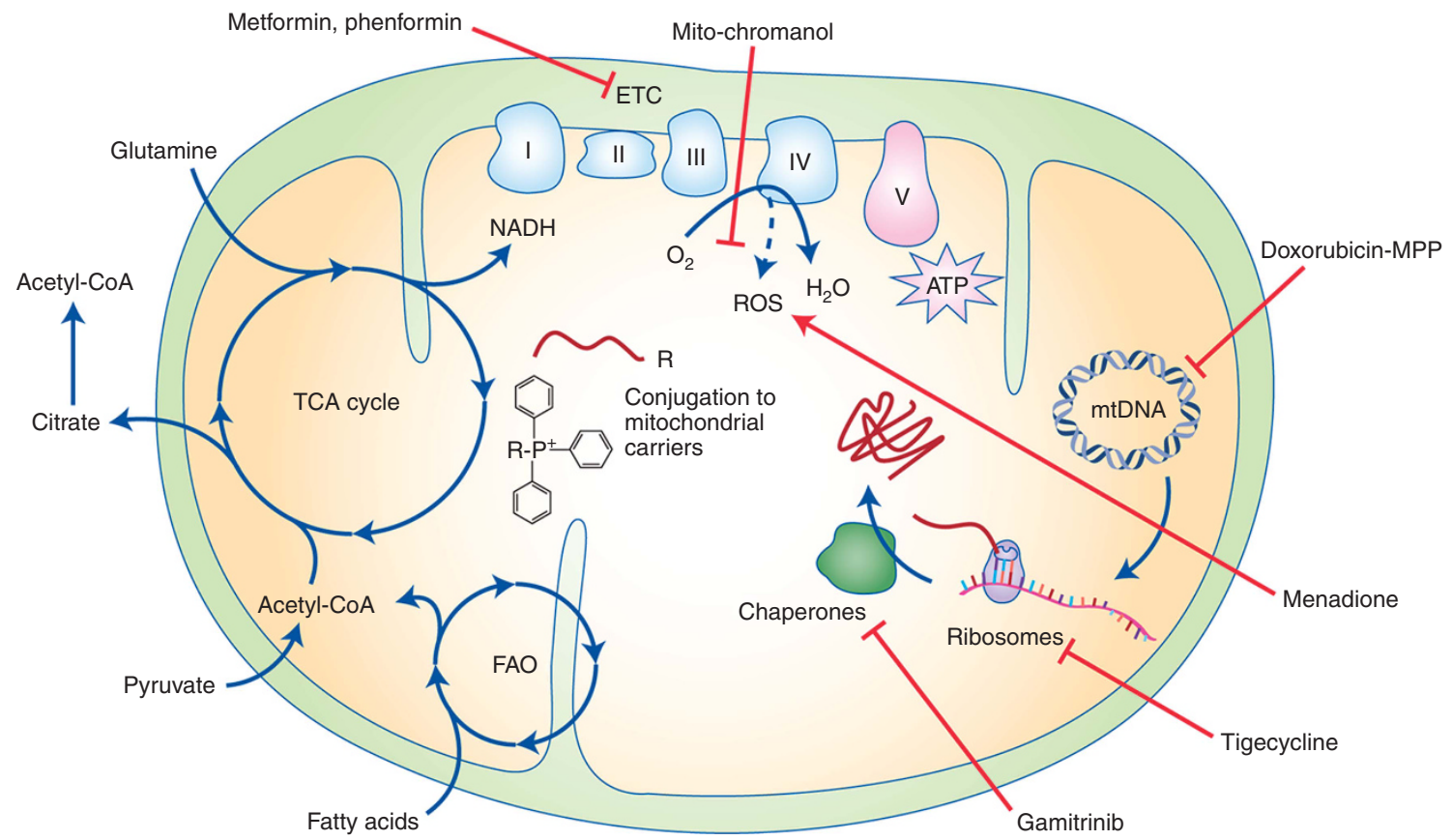

Figure 2. Targeting cancer stem cells through inhibition of mitochondrial function. CSCs dependent of OXPHOS can be eliminated by various strategies impairing mitochondrial energy metabolism. Direct inhibition of OXPHOS can be achieved with small molecules such as the antidiabetic agents metformin and phenformin, which inhibit the ETC Complex I and cause cell death by energy crisis in CSCs. Conjugation of pharmacologic agents to mitochondrial carriers such as TPP or mitochondria-penetrating peptides (MPPs) may allow their selective delivery and accumulation in mitochondria. This strategy has already been used for chemotherapeutic agents such as doxorubicin to selectively disrupt mtDNA integrity and the expression of ETC proteins. Mitochondrial protein biosynthesis can also be blocked by the inhibition of mitochondrial ribosomes using tigecycline and other FDA-approved antibiotics, which impair OXPHOS and bear toxicity against CSCs. Similarly, the functionality of ETC components can also be targeted by mitochondrial delivery of the chaperone inhibitor gamitrinib. Cell signalling by OXPHOS-generated mitochondrial ROS is crucial for cancer cell proliferation and can be targeted by the mitochondrial accumulation of antioxidants such as mito-chromanol. Conversely, CSCs can be eliminated by inducing toxic levels of ROS in mitochondria with the ROS-inducer menadione. Finally, OXPHOS can also be impaired at the level of mitochondrial carbon metabolism, either by altering the enzymes involved in the TCA cycle or fatty acid oxidation (FAO) or by interfering with the supply of mitochondrial fuels.

As mitochondria originally evolved from bacteria, it is not surprising that multiple antibiotics can disrupt mitochondrial function. Indeed, a recent study suggested that the self-renewal capacity in multiple tumour types could be targeted by treatment with certain widely prescribed antibiotics via disrupting mitochondrial respiration, either by the inhibition of mitochondrial ribosomes or by the direct targeting of OXPHOS (Lamb et al, 2015b, c).

The antidiabetic agent metformin has also emerged as a promising candidate for targeting OXPHOS in pancreatic sphere-forming and $\mathrm{CD}_{13}{ }^{+}$cells, respectively (Sancho et al, 2015). Interest in metformin emerged after retrospective studies suggested a lower incidence of cancer in diabetic patients treated with metformin compared with other antidiabetic regimens. Although the reduced insulin levels may also contribute to the reported effects, compelling evidence demonstrated that the antitumoural activity of metformin involved the impairment of OXPHOS via direct inhibition of mitochondrial Complex I (Wheaton et al, 2014). Interestingly, metformin selectively induced apoptosis in pancreatic $\mathrm{CD} 133^{+}$cells as a result of their inability to switch to glycolysis and subsequent energy crisis (Sancho et al, 2015). Still, a minor subset of $\mathrm{CD}_{133^{+}}$cells displaying low mitochondrial mass and a predominantly glycolytic metabolism were inherently resistant to metformin. The presence of these cells in PDAC appeared to account for the observed uniform relapse of tumours in mice treated with metformin and may provide an alternative rational for the negative outcome of the first clinical trials testing the effects of metformin in PDAC patients (Kordes et al, 2015), while, based on recent pharmacokinetics data (Chandel et al, 2016; Dowling et al, 2016; Sivalingam et al, 2016), the originally quoted insufficient bioavailability of metformin seems less likely to be a major contributing factor.

These data suggest that metformin and other drugs targeting mitochondrial ATP production need to be combined with agents counteracting the mechanism of resistance allowing some CSCs to overcome OXPHOS inhibition, for example, via modulation of the PGC1- $\alpha / \mathrm{MYC}$ ratio. Intriguingly, metformin resistance in pancreatic $\mathrm{CD}_{133^{+}}$cells could be prevented/reversed by the knockdown of MYC expression using shRNA or indirectly via bromodomain and extra-terminal motif (BET) inhibition, suggesting the therapeutic potential of MYC inhibition in combination with mitochondrial targeting for PDAC treatment (Sancho et al, 2015). Alternatively, agents interfering with mitochondrial function at various levels may be more effective in targeting all CSCs. In fact, while drug resistance was observed with most OXPHOS inhibitors, such as rotenone or resveratrol, resistance was not observed for menadione, a drug which acts via dual mechanism - inhibition of Complex I and induction of mitochondrial ROS. On the other hand, the efficacy of metformin may also be limited by its requirement of organic cation transporters (OCTs) for cellular uptake, which restricts its effects in healthy tissues but also limits its potential use to tumour cells expressing OCTs. Phenformin, another biguadine formerly used in diabetes, could overcome this limitation as it is more hydrophobic and is delivered to mitochondria more efficiently than metformin. Phenformin also promotes cancer cell death by inhibiting Complex I and has offered promising 
preclinical results in certain cancers such as non-small-cell lung carcinoma (Shackelford et al, 2013).

Therefore, an important factor concerning the design of novel pharmacological strategies targeting mitochondria is to ensure the efficient and preferably selective delivery of the drug to the mitochondria in cancer cells. CSCs relying on OXPHOS show an elevated mitochondrial membrane potential $(\Delta \psi \mathrm{m})$, which can be exploited for selectively increasing drug delivery to the mitochondria of these cells. Delocalised lipophilic cations such as triphenylphosphonium (TPP) accumulate in the mitochondrial matrix and can be conjugated to small compounds for selective drug delivery to mitochondria (Murphy, 2008). The mitochondrial accumulation of mito-chromanol, a vitamin $\mathrm{E}$ analogue conjugated to TPP, induced cell death by inhibiting OXPHOS in breast cancer cells without affecting non-transformed cells (Cheng et al, 2013). Conjugation with TPP has also been utilised to selectively deliver the chaperone inhibitor gamitrinib to active mitochondria and disrupt energy production in cancer cells by impairing protein folding in mitochondria (Chae et al, 2012). However, mitochondria-penetrating peptides might be preferred for the treatment of certain tumours, as they can deliver cargo molecules irrespective of $\Delta \psi \mathrm{m}$. Their conjugation to chemotherapeutic agents, such as doxorubicin, directs their activity towards mitochondrial DNA (mtDNA), promoting drug selectivity for cancer cells with reduced mtDNA integrity while their stable mitochondrial localisation prevents the acquisition of resistance by drug efflux (Chamberlain et al, 2013).

\section{CONCLUSIONS}

In various cancers, CSCs have now been shown to bear a distinct metabolic phenotype and are highly dependent on OXPHOS and/or mitochondrial function. The reasons are likely multifactorial. Apart from gaining greater independence from the sparse nutritional support in the tumour microenvironment, increasing evidence now also suggests that the tight control of mitochondrial ROS production in CSCs is a prerequisite for maintaining their stemness and high fidelity. This apparent metabolic vulnerability provides vast new therapeutic opportunities to more efficiently eliminate these highly tumourigenic cells, even though resistance may arise in some instances (Sancho et al, 2015). The latter could be related either to the acquisition of metabolic plasticity in a subset of CSCs or to the pre-existence of a small subset of CSCs with a higher degree of metabolic plasticity. As the latter bear reduced stemness properties, they are less likely to significantly contribute to cancer progression in treatment naive tumours, but metabolic targeting may provide these cells with a selective advantage so that they eventually take over and drive relapse of the disease. Thus, a more effective strategy could be to combine distinct targeting strategies or to use mitochondria-targeting agents with dual mechanism of action. Efficient metabolic targeting of all CSCs may help to eventually improve the still poor outcome of many cancer patients.

\section{ACKNOWLEDGEMENTS}

This work was supported by the ERC Advanced Investigator Grant (Pa-CSC 233460 to $\mathrm{CH}$ ), the European Community's Seventh Framework Programme (FP7/2007-2013) under grant agreement no 256974 (EPC-TM-NET to C.H.) and no 602783 (CAM-PaC to C.H.), the 2015 SU2C Lustgarten CRUK Pancreatic Cancer Dream Team Award (to CH), Pancreatic Cancer UK RIF2014_04 and RIF2015_03 (both to $\mathrm{CH}$ ) and the Pancreatic Cancer Research Fund (to PS).

\section{CONFLICT OF INTEREST}

The authors declare no conflict of interest.

\section{REFERENCES}

Anderson AS, Roberts PC, Frisard MI, Hulver MW, Schmelz EM (2014) Ovarian tumor-initiating cells display a flexible metabolism. Exp Cell Res 328(1): 44-57.

Carracedo A, Cantley LC, Pandolfi PP (2013) Cancer metabolism: fatty acid oxidation in the limelight. Nat Rev Cancer 13(4): 227-232.

Carracedo A, Weiss D, Leliaert AK, Bhasin M, de Boer VC, Laurent G, Adams AC, Sundvall M, Song SJ, Ito K, Finley LS, Egia A, Libermann T, Gerhart-Hines Z, Puigserver P, Haigis MC, Maratos-Flier E, Richardson AL, Schafer ZT, Pandolfi PP (2012) A metabolic prosurvival role for PML in breast cancer. J Clin Invest 122(9): 3088-3100.

Chae YC, Caino MC, Lisanti S, Ghosh JC, Dohi T, Danial NN, Villanueva J, Ferrero S, Vaira V, Santambrogio L, Bosari S, Languino LR, Herlyn M, Altieri DC (2012) Control of tumor bioenergetics and survival stress signaling by mitochondrial HSP90s. Cancer Cell 22(3): 331-344.

Chamberlain GR, Tulumello DV, Kelley SO (2013) Targeted delivery of doxorubicin to mitochondria. ACS Chem Biol 8(7): 1389-1395.

Chandel NS (2014) Mitochondria as signaling organelles. BMC Biol 12: 34.

Chandel NS, Avizonis D, Reczek CR, Weinberg SE, Menz S, Neuhaus R, Christian S, Haegebarth A, Algire C, Pollak M (2016) Are metformin doses used in murine cancer models clinically relevant? Cell Metab 23(4): 569-570.

Chen CL, Uthaya Kumar DB, Punj V, Xu J, Sher L, Tahara SM, Hess S, Machida K (2015) NANOG metabolically reprograms tumor-initiating stem-like cells through tumorigenic changes in oxidative phosphorylation and fatty acid metabolism. Cell Metab 23(1): 206-219.

Chen CL, Uthaya Kumar DB, Punj V, Xu J, Sher L, Tahara SM, Hess S, Machida K (2016) NANOG metabolically reprograms tumor-initiating stem-like cells through tumorigenic changes in oxidative phosphorylation and fatty acid metabolism. Cell Metab 23(1): 206-219.

Cheng G, Zielonka J, McAllister DM, Mackinnon Jr AC, Joseph J, Dwinell MB, Kalyanaraman B (2013) Mitochondria-targeted vitamin E analogs inhibit breast cancer cell energy metabolism and promote cell death. BMC Cancer 13: 285.

Clausell-Tormos J, Azevedo MM, Miranda-Lorenzo I, Vieira CR, Sanchez-Ripoll Y, Megias D, Heeschen C (2014) Nano-volume well array chip for large-scale propagation and high-resolution analysis of individual cancer stem cells. J Nanomed Nanotechnol 5(191): 1000191-1000198.

Davidson SM, Papagiannakopoulos T, Olenchock BA, Heyman JE, Keibler MA, Luengo A, Bauer MR, Jha AK, O’Brien JP, Pierce KA, Gui DY, Sullivan LB, Wasylenko TM, Subbaraj L, Chin CR, Stephanopolous G, Mott BT, Jacks T, Clish CB, Vander Heiden MG (2016) Environment impacts the metabolic dependencies of Ras-driven non-small cell lung cancer. Cell Metab 23(3): 517-528.

De Luca A, Fiorillo M, Peiris-Pages M, Ozsvari B, Smith DL, Sanchez-Alvarez R, Martinez-Outschoorn UE, Cappello AR, Pezzi V, Lisanti MP, Sotgia F (2015) Mitochondrial biogenesis is required for the anchorageindependent survival and propagation of stem-like cancer cells. Oncotarget 6(17): 14777-14795.

Diehn M, Cho RW, Lobo NA, Kalisky T, Dorie MJ, Kulp AN, Qian D, Lam JS, Ailles LE, Wong M, Joshua B, Kaplan MJ, Wapnir I, Dirbas FM, Somlo G, Garberoglio C, Paz B, Shen J, Lau SK, Quake SR, Brown JM, Weissman IL, Clarke MF (2009) Association of reactive oxygen species levels and radioresistance in cancer stem cells. Nature 458(7239): 780-783.

Dong C, Yuan T, Wu Y, Wang Y, Fan TW, Miriyala S, Lin Y, Yao J, Shi J, Kang T, Lorkiewicz St P, Clair D, Hung MC, Evers BM, Zhou BP (2013) Loss of FBP1 by Snail-mediated repression provides metabolic advantages in basal-like breast cancer. Cancer Cell 23(3): 316-331.

Dowling RJ, Lam S, Bassi C, Mouaaz S, Aman A, Kiyota T, Al-Awar R, Goodwin PJ, Stambolic V (2016) Metformin pharmacokinetics in mouse tumors: implications for human therapy. Cell Metab 23(4): 567-568.

Farnie G, Sotgia F, Lisanti MP (2015) High mitochondrial mass identifies a sub-population of stem-like cancer cells that are chemo-resistant. Oncotarget 6(31): 30472-30486.

Folmes CD, Dzeja PP, Nelson TJ, Terzic A (2012) Metabolic plasticity in stem cell homeostasis and differentiation. Cell Stem Cell 11(5): 596-606. 
Folmes CD, Martinez-Fernandez A, Faustino RS, Yamada S, Perez-Terzic C, Nelson TJ, Terzic A (2013) Nuclear reprogramming with c-Myc potentiates glycolytic capacity of derived induced pluripotent stem cells. J Cardiovasc Transl Res 6(1): 10-21.

Gabay M, Li Y, Felsher DW (2014) MYC activation is a hallmark of cancer initiation and maintenance. Cold Spring Harb Perspect Med 4(6): a014241.

Garcia-Silva S, Frias-Aldeguer J, Heeschen C (2013) Stem cells \& pancreatic cancer. Pancreatology 13(2): 110-113.

Gupta PB, Onder TT, Jiang G, Tao K, Kuperwasser C, Weinberg RA, Lander ES (2009) Identification of selective inhibitors of cancer stem cells by high-throughput screening. Cell 138(4): 645-659.

Hermann PC, Huber SL, Herrler T, Aicher A, Ellwart JW, Guba M, Bruns CJ, Heeschen C (2007) Distinct populations of cancer stem cells determine tumor growth and metastatic activity in human pancreatic cancer. Cell Stem Cell 1(3): 313-323.

Ito K, Carracedo A, Weiss D, Arai F, Ala U, Avigan DE, Schafer ZT, Evans RM, Suda T, Lee CH, Pandolfi PP (2012) A PML-PPAR-delta pathway for fatty acid oxidation regulates hematopoietic stem cell maintenance. Nat Med 18(9): 1350-1358.

Janiszewska M, Suva ML, Riggi N, Houtkooper RH, Auwerx J, Clement-Schatlo V, Radovanovic I, Rheinbay E, Provero P, Stamenkovic I (2012) Imp2 controls oxidative phosphorylation and is crucial for preserving glioblastoma cancer stem cells. Genes Dev 26(17): 1926-1944.

Kordes S, Pollak MN, Zwinderman AH, Mathot RA, Weterman MJ, Beeker A, Punt CJ, Richel DJ, Wilmink JW (2015) Metformin in patients with advanced pancreatic cancer: a double-blind, randomised, placebocontrolled phase 2 trial. Lancet Oncol 16(7): 839-847.

Lagadinou ED, Sach A, Callahan K, Rossi RM, Neering SJ, Minhajuddin M, Ashton JM, Pei S, Grose V, O’Dwyer KM, Liesveld JL, Brookes PS, Becker MW, Jordan CT (2013) BCL-2 inhibition targets oxidative phosphorylation and selectively eradicates quiescent human leukemia stem cells. Cell Stem Cell 12(3): 329-341.

Lamb R, Bonuccelli G, Ozsvari B, Peiris-Pages M, Fiorillo M, Smith DL, Bevilacqua G, Mazzanti CM, McDonnell LA, Naccarato AG, Chiu M, Wynne L, Martinez-Outschoorn UE, Sotgia F, Lisanti MP (2015a) Mitochondrial mass, a new metabolic biomarker for stem-like cancer cells: Understanding WNT/FGF-driven anabolic signaling. Oncotarget 6(31): 30453-30471.

Lamb R, Fiorillo M, Chadwick A, Ozsvari B, Reeves KJ, Smith DL, Clarke RB, Howell SJ, Cappello AR, Martinez-Outschoorn UE, Peiris-Pages M, Sotgia F, Lisanti MP (2015b) Doxycycline down-regulates DNA-PK and radiosensitizes tumor initiating cells: Implications for more effective radiation therapy. Oncotarget 6(16): 14005-14025.

Lamb R, Harrison H, Hulit J, Smith DL, Lisanti MP, Sotgia F (2014) Mitochondria as new therapeutic targets for eradicating cancer stem cells: quantitative proteomics and functional validation via MCT1/2 inhibition. Oncotarget 5(22): 11029-11037.

Lamb R, Ozsvari B, Lisanti CL, Tanowitz HB, Howell A, Martinez-Outschoorn UE, Sotgia F, Lisanti MP (2015c) Antibiotics that target mitochondria effectively eradicate cancer stem cells, across multiple tumor types: treating cancer like an infectious disease. Oncotarget 6(7): $4569-4584$

Lonardo E, Hermann PC, Mueller MT, Huber S, Balic A, Miranda-Lorenzo I, Zagorac S, Alcala S, Rodriguez-Arabaolaza I, Ramirez JC, Torres-Ruiz R, Garcia E, Hidalgo M, Cebrian DA, Heuchel R, Lohr M, Berger F, Bartenstein P, Aicher A, Heeschen C (2011) Nodal/Activin signaling drives self-renewal and tumorigenicity of pancreatic cancer stem cells and provides a target for combined drug therapy. Cell Stem Cell 9(5): 433-446.

Malanchi I, Santamaria-Martinez A, Susanto E, Peng H, Lehr HA, Delaloye JF, Huelsken J (2012) Interactions between cancer stem cells and their niche govern metastatic colonization. Nature 481(7379): 85-89.

Martinez-Outschoorn UE, Prisco M, Ertel A, Tsirigos A, Lin Z, Pavlides S, Wang C, Flomenberg N, Knudsen ES, Howell A, Pestell RG, Sotgia F, Lisanti MP (2011) Ketones and lactate increase cancer cell "stemness," driving recurrence, metastasis and poor clinical outcome in breast cancer: achieving personalized medicine via Metabolo-Genomics. Cell Cycle 10(8): 1271-1286.

Miranda-Lorenzo I, Dorado J, Lonardo E, Alcala S, Serrano AG, Clausell-Tormos J, Cioffi M, Megias D, Zagorac S, Balic A, Hidalgo M, Erkan M, Kleeff J, Scarpa A, Sainz Jr B, Heeschen C (2014) Intracellular autofluorescence: a biomarker for epithelial cancer stem cells. Nat Methods 11(11): 1161-1169.
Mullen AR, Wheaton WW, Jin ES, Chen PH, Sullivan LB, Cheng T, Yang Y, Linehan WM, Chandel NS, DeBerardinis RJ (2012) Reductive carboxylation supports growth in tumour cells with defective mitochondria. Nature 481(7381): 385-388.

Murphy MP (2008) Targeting lipophilic cations to mitochondria. Biochim Biophys Acta 1777(7-8): 1028-1031.

Nakajima EC, Van Houten B (2013) Metabolic symbiosis in cancer: refocusing the Warburg lens. Mol Carcinog 52(5): 329-337.

Pasto A, Bellio C, Pilotto G, Ciminale V, Silic-Benussi M, Guzzo G, Rasola A, Frasson C, Nardo G, Zulato E, Nicoletto MO, Manicone M, Indraccolo S, Amadori A (2014) Cancer stem cells from epithelial ovarian cancer patients privilege oxidative phosphorylation, and resist glucose deprivation. Oncotarget 5(12): 4305-4319.

Sainz Jr B, Alcala S, Garcia E, Sanchez-Ripoll Y, Azevedo MM, Cioffi M, Tatari M, Miranda-Lorenzo I, Hidalgo M, Gomez-Lopez G, Canamero M, Erkan M, Kleeff J, Garcia-Silva S, Sancho P, Hermann PC, Heeschen C (2015) Microenvironmental hCAP-18/LL-37 promotes pancreatic ductal adenocarcinoma by activating its cancer stem cell compartment. Gut 64(12): 1921-1935.

Sainz Jr B, Martin B, Tatari M, Heeschen C, Guerra S (2014) ISG15 is a critical microenvironmental factor for pancreatic cancer stem cells. Cancer Res 74(24): 7309-7320.

Samudio I, Harmancey R, Fiegl M, Kantarjian H, Konopleva M, Korchin B, Kaluarachchi K, Bornmann W, Duvvuri S, Taegtmeyer H, Andreeff M (2010) Pharmacologic inhibition of fatty acid oxidation sensitizes human leukemia cells to apoptosis induction. J Clin Invest 120(1): 142-156.

Sancho P, Burgos-Ramos E, Tavera A, Bou Kheir T, Jagust P, Schoenhals M, Barneda D, Sellers K, Campos-Olivas R, Grana O, Viera CR, Yuneva M, Sainz Jr B, Heeschen C (2015) MYC/PGC-1a balance determines the metabolic phenotype and plasticity of pancreatic cancer stem cells. Cell Metab 22(4): 590-605.

Schafer ZT, Grassian AR, Song L, Jiang Z, Gerhart-Hines Z, Irie HY, Gao S, Puigserver P, Brugge JS (2009) Antioxidant and oncogene rescue of metabolic defects caused by loss of matrix attachment. Nature 461(7260): 109-113.

Senkowski W, Zhang X, Olofsson MH, Isacson R, Hoglund U, Gustafsson M, Nygren P, Linder S, Larsson R, Fryknas M (2015) Three-dimensional cell culture-based screening identifies the anthelmintic drug nitazoxanide as a candidate for treatment of colorectal cancer. Mol Cancer Ther 14(6): 1504-1516.

Shackelford DB, Abt E, Gerken L, Vasquez DS, Seki A, Leblanc M, Wei L, Fishbein MC, Czernin J, Mischel PS, Shaw RJ (2013) LKB1 inactivation dictates therapeutic response of non-small cell lung cancer to the metabolism drug phenformin. Cancer cell 23(2): 143-158.

Shen YA, Lin CH, Chi WH, Wang CY, Hsieh YT, Wei YH, Chen YJ (2013) Resveratrol impedes the stemness, epithelial-mesenchymal transition, and metabolic reprogramming of cancer stem cells in nasopharyngeal carcinoma through p53 activation. Evid Based Complement Alternat Med 2013: 590393.

Shen YA, Wang CY, Hsieh YT, Chen YJ, Wei YH (2015) Metabolic reprogramming orchestrates cancer stem cell properties in nasopharyngeal carcinoma. Cell Cycle 14(1): 86-98.

Sivalingam VN, Kitson S, McVey R, Roberts C, Pemberton P, Gilmour K, Ali S, Renehan AG, Kitchener HC, Crosbie EJ (2016) Measuring the biological effect of presurgical metformin treatment in endometrial cancer. Br J Cancer 114(3): 281-289.

Skrtic M, Sriskanthadevan S, Jhas B, Gebbia M, Wang X, Wang Z, Hurren R, Jitkova Y, Gronda M, Maclean N, Lai CK, Eberhard Y, Bartoszko J, Spagnuolo P, Rutledge AC, Datti A, Ketela T, Moffat J, Robinson BH, Cameron JH, Wrana J, Eaves CJ, Minden MD, Wang JC, Dick JE, Humphries K, Nislow C, Giaever G, Schimmer AD (2011) Inhibition of mitochondrial translation as a therapeutic strategy for human acute myeloid leukemia. Cancer Cell 20(5): 674-688.

Vander Heiden MG, Cantley LC, Thompson CB (2009) Understanding the Warburg effect: the metabolic requirements of cell proliferation. Science 324(5930): 1029-1033.

Vazquez-Martin A, Oliveras-Ferraros C, Cufi S, Del Barco S, Martin-Castillo B, Menendez JA (2010) Metformin regulates breast cancer stem cell ontogeny by transcriptional regulation of the epithelial-mesenchymal transition (EMT) status. Cell Cycle 9(18): 3807-3814.

Visvader JE, Lindeman GJ (2012) Cancer stem cells: current status and evolving complexities. Cell Stem Cell 10(6): 717-728. 
Wang YC, Chao TK, Chang CC, Yo YT, Yu MH, Lai HC (2013) Drug screening identifies niclosamide as an inhibitor of breast cancer stem-like cells. PLoS One 8(9): e74538.

Wheaton WW, Weinberg SE, Hamanaka RB, Soberanes S, Sullivan LB, Anso E, Glasauer A, Dufour E, Mutlu GM, Budigner GS, Chandel NS (2014) Metformin inhibits mitochondrial complex I of cancer cells to reduce tumorigenesis. Elife 3: e02242.

Yang MH, Imrali A, Heeschen C (2015) Circulating cancer stem cells: the importance to select. Chin J Cancer Res 27(5): 437-449.

Ye XQ, Li Q, Wang GH, Sun FF, Huang GJ, Bian XW, Yu SC, Qian GS (2011) Mitochondrial and energy metabolism-related properties as novel indicators of lung cancer stem cells. Int J Cancer 129(4): 820-831.

Yo YT, Lin YW, Wang YC, Balch C, Huang RL, Chan MW, Sytwu HK, Chen CK, Chang CC, Nephew KP, Huang T, Yu MH, Lai HC (2012) Growth inhibition of ovarian tumor-initiating cells by niclosamide. Mol Cancer Ther 11(8): 1703-1712.

(c) (1) (2) This work is licensed under the Creative Commons At ${ }_{\text {BY }}$ SA Atribution-Non-Commercial-Share Alike 4.0 International License. To view a copy of this license, visit http:// creativecommons.org/licenses/by-nc-sa/4.0/ 